\title{
Administration of medicines by emergency nurse practitioners according to protocols in an accident and emergency department
}

\author{
Julie Marshall, Carol Edwards, Mike Lambert
}

\begin{abstract}
Objective-To present the legal and professional issues related to nurse administration of drugs according to protocols, and describe the implementation and initial audit findings of such a scheme. Setting-Accident and emergency (A\&E) department of a district general hospital. Methods-Analysis of legal and professional opinion. Protocols acceptable to the medical, nursing, and pharmacy professions were developed across a wide range of drugs appropriate for administration by accident and emergency nurse practitioners (ENPs). The first six months of the scheme were audited. Audit initially addressed general compliance with protocols and later the specific areas of tetanus immunisation and emergency contraception.

Results-ENPs assessed 2925 patients in six months (10.9\% of all new patients); 455 patients (15.5\% of the ENP patients) were given drugs according to protocols. There were no breaches of the protocols. Subsequent audit of tetanus immunisation showed 94-100\% compliance with protocol standards and $\mathbf{7 1 - 1 0 0 \%}$ compliance for emergency contraception.

Conclusions-There are no legal or professional obstacles to the development of protocols for the administration of drugs to patients by nurses without reference to a doctor, providing the protocols meet all the requirements of the UKCC and have the support of consultant medical staff. Such a system must be subject to regular audit to promote a dynamic approach to protocols and training. The system safely enhanced the quality of care of patients treated by ENPs in A\&E.

( $尹$ Accid Emerg Med 1997;14:233-237)
\end{abstract}

Nursing Practice

(Education and

Development), Norfolk and Norwich Hospital, Norwich

C Edwards

Correspondence to: Mr M A Lambert, Consultant in Accident and Emergency Medicine, Accident and Emergency Department, Norfolk and Department, Norfolk and NR1 3SR.

Keywords: accident and emergency; drug administration; emergency nurse practitioners

Emergency nurse practitioners (ENPs) were introduced to the accident and emergency (A\&E) department of the Norfolk \& Norwich Hospital in February 1995. The role of the ENP is to assess, diagnose, treat, and advise patients within defined protocols. We felt the service would be enhanced if the ENPs gave appropriate medication to patients without the need to consult a doctor. We hoped this would result in improved patient satisfaction and ENP job satisfaction.

In December 1995 a working party was formed to address this issue. The working party reviewed the legal and professional issues surrounding the development of nurse administration of drugs. Its recommendations were then submitted to the Trust Board.

This paper outlines our interpretation of the legal and professional issues, how the administration of drugs by ENPs according to clinical protocols was developed and implemented, and our experience of audit of the administration of drugs against these protocols.

\section{Legal issues}

The working party obtained written opinion from the United Kingdom Central Council for Nursing, Midwifery and Health Visiting (UKCC), the Royal Pharmaceutical Society of Great Britain, the General Medical Council (GMC), and the Medical Defence Union (MDU).

Section 58 (2) of the Medicines Act $1968^{1}$ sets out the relevant statutory position regarding nurses giving medicines to patients which have not been directly prescribed for a named patient by a doctor. This section of the Act states that "prescription only medicines" should only be given by "an appropriate practitioner, ie a doctor, a dentist, or a vet, or $a$ person acting in accordance with the directions of an appropriate practitioner".

From the various opinions it was understood that "directions" may be verbal or written (as in a prescription), and that a protocol may constitute "directions". We concluded that an emergency nurse practitioner, acting in accordance with a protocol, could give prescription-only medicines without that protocol referring to a specific patient.

\section{Professional issues}

There are five key professional issues which affect the role of nurses giving medicines:

(1) UKCC code of professional conduct ${ }^{2}$

(2) Standards for the administration of medicines ${ }^{3}$

(3) The scope of professional practice ${ }^{4}$

(4) UKCC, Rule $18(1)^{5}$

(5) Exercising accountability. ${ }^{6}$

The UKCC produced Standards for the administration of medicines in October 1992. Paragraph 6.2 sets out the Council's view on the administration of medicines to patients. It 
states that "Where it is the wish of the professional staff concerned that practitioners in a particular setting be authorised to administer, on their own authority, certain medicines, a local protocol [should be] agreed between medical practitioners, nurses and midwives and the pharmacist." 3

The UKCC also states that “... in any situation in which a practitioner may be expected or required to administer 'prescription only medicines' which have not been directly prescribed for a named patient by a registered medical practitioner who has examined the patient and made a diagnosis, it is essential that a clear local policy be determined and made known to all practitioners involved with prescribing and administration. This will make it possible for action to be taken in patients' interests while protecting practitioners from risk of complaint that might otherwise jeopardise their position" (Para 35). ${ }^{3}$ It is further stated that a local policy should be agreed and documented which makes clear the circumstances under which a particular 'prescription-only medicine' may be given, ensures the relevant knowledge and skill of those to be involved in administration, describes the form, route, and dose range of the medicines so authorised, and satisfies the requirements of section 58 of the Medicines Act 1968 as a "Direction".

Rule 18 (1) of the Nurses, Midwives and Health Visitors Rules Approval Order No 873, states that successful "admission to Part 1, 3, 5 or 8 of the register entails accepting responsibility to be able to assess, plan, implement and evaluate care, whereas admission to Part $2,4,6$ entails the nurse being able to plan and implement nursing care". This means that a first level registered nurse, rather than enrolled nurses, could undertake this role, as the nurse must be able to assess and evaluate the care of the patient before and after administering medicines. The UKCC strengthens this position by maintaining that a first level registered nurse "must endeavour always to achieve, maintain and develop knowledge, skill and competence to respond to those needs and interests" and "must honestly acknowledge any limits of personal knowledge and skill and take steps to remedy any relevant deficits in order effectively and appropriately to meet the needs of patients and clients." (Para 9.2, 9.3.)

Nurses giving medicines are also required to have an understanding of the substances used for therapeutic purposes, be able to justify any actions they undertake, and be prepared to be accountable for any action taken.

In the exercise of this professional accountability, the UKCC Code of Professional Conduct $^{2}$ insists that nurses must: (1) act always in such a manner as to promote and safeguard the interests and wellbeing of patients; (2) ensure that no action or omission on their part, or within their sphere of responsibility, is detrimental to the interests, condition, or safety of patients and clients; (3) maintain and improve their professional knowledge and competence; (4) acknowledge any limitations in their knowledge and competence and decline any duties or responsibilities unless able to perform them in a safe and skilled manner.

We therefore concluded that the UKCC recognised that appropriate protocols could be developed to support a nurse giving drugs to a patient, even though that patient had not been assessed or diagnosed by a registered medical practitioner, if all the above conditions and requirements were met. The development and implementation of such a protocol would have to recognise the nurses' responsibilities under the Scope of professional practice. ${ }^{4}$

\section{MEDICAL}

It was important to determine the accountability of medical staff who were clinically responsible for a patient who may receive medication according to protocols. Advice was taken from the GMC and the MDU. It was accepted that a doctor, while remaining clinically responsible for a patient, is entitled to delegate a task to someone without medical qualifications. The GMC guidance to doctors makes it clear that "a doctor who delegates treatment or other procedures must be satisfied that the person to whom they are delegated is competent to carry them out", and "that the doctor should retain ultimate responsibility for the management of their patients because only the doctor has received the necessary training to undertake this responsibility."7

We were advised that a consultant proposing to develop protocols for the administration of drugs by a nurse must be satisfied that the nurse has the appropriate training, skills, and competence to provide the standard of care expected of an experienced nurse giving medicines within that clinical area. Therefore, providing a consultant is involved in the development of protocols and is satisfied that nurses who will be giving the drug according to that protocol have the appropriate training and experience, there was no professional or medicolegal objection to the development of protocols.

\section{PHARMACEUTICAL}

Advice from the law department of the Royal Pharmaceutical Society of Great Britain made it clear that the Medicines Act of 1968 did not prohibit the setting of written protocols for the administration/supply of medicines by nurses. It was also stated that protocols could be developed for both inpatients and outpatients of the hospital.

\section{CONCLUSIONS}

Based on analysis of the legal and professional issues the working party concluded that nurses could administer drugs to patients according to a clinical protocol, without the doctors, nurses or pharmacists being in breach of the law or professional obligations providing certain criteria were met. These criteria included the format and contents of the protocol, and arrangements for training, accreditation and audit. 
Clinical protocols

We advised that a clinical protocol for the administration of drugs must include:

- a rationale for the administration of the drug, including a nursing assessment of the patient;

- the circumstances under which the drug will be given;

- the form of the drug to be given, for example tablet, injection, etc;

- the route by which the drug will be given;

- the dose of the drug to be given, including the number of doses or length of course;

- instructions regarding drug contraindications, precautions, and side effect profile;

- instructions to check for allergies;

- instruction to check for concomitant medication and for drug interactions.

Since consultants remain clinically responsible for a patients they must be actively involved in the preparation of protocols and satisfy themselves that the nurses who would give the drugs had sufficient understanding, knowledge, and skills relevant to give that drug. It was therefore expected that formal training programmes, supervised by the consultants, would be developed.

Our overall conclusion was that if all these requirements were met there was no legal or professional obstacle to the implementation of clinical protocols for the administration of drugs to patients by nurses without direct reference to a doctor.

\section{Implementation}

The report of the Trust working party was submitted to the drugs and therapeutics committee and subsequently to the Trust Board through the executive director for nursing and human resources. After consultation with the Trust solicitors the recommendations were accepted with the proviso that all protocol development should be submitted to the drugs and therapeutics committee for approval before implementation. The Trust accepted vicarious liability on behalf of the nurses following the protocols.

DEVELOPMENT OF PROTOCOLS

Within the A\&E department there were discussions between ENPs, consultants, and pharmacists to identify groups of drugs which would be useful to the ENPs, and to identify the specific drugs that could be made available. These discussions identified simple analgesics, non-steroidal anti-inflammatory drugs, antihistamines, antibiotics, postcoital contraception, tetanus immunisation, and local anaesthesia as useful and appropriate for ENPs to administer (table 1 ).

Protocols which met all the criteria were developed, with the $A \& E$ consultants, by the ENPs.

\section{TRAINING}

To be eligible for the ENP role we required a minimum of three years of full time $A \& E$ nursing experience, plus successful completion of an appropriate course. It was agreed that for ENPs to be accredited by the Trust to give
Table 1 Drugs for ENP administration according to protocol

\begin{tabular}{ll}
\hline Paracetamol & Flucloxacillin \\
CoDydramol & Chlorpheniramine \\
Ibuprofen & Loratadine \\
Diclofenac & PC-4 \\
Erythromycin & Tetanus toxoid \\
Penicillin & Tetanus immunoglobulin \\
Co-amoxiclav & Lignocaine hydrochloride \\
\hline
\end{tabular}

drugs, and be acceptable to the consultants in accident and emergency medicine who are ultimately responsible for patient care, they should undergo additional training. All the ENPs attended a study day on which the consultants gave lectures on the basic pharmacology of the drugs and their use within the protocol. The aim of these training sessions was to give the ENPs sufficient understanding of the drugs to ensure that they would be given in a "safe and skilled manner."

Following the study day, the ENPs were accredited by the department and Trust to give certain drugs according to protocols.

Six nurses were trained as ENPs with authority to give drugs. None of the nurses acts as a full time ENP. Their general accident and emergency and ENP skills are used flexibly and appropriately, depending on the demands on the A\&E department.

It was agreed that there would be an annual review of the protocols with a further study day and reaccreditation to ensure that the protocols remain appropriate for our A\&E department. The consultants and ENPs also undertook to monitor changes in practice and pharmaceutical developments so that the protocols and available drugs could be revised as necessary. ENPs started the administration of drugs according to protocols in February 1996.

\section{Audit}

It was felt that audit of this innovative system was essential. Initially individual ENPs had all decisions to give drugs reviewed against the departmental protocols by another ENP. In addition audit was undertaken of approximately one in 10 of the ENP cases in which no drugs had been given, to monitor whether patients who would have benefited from the administration of drugs were not receiving them.

In the first six months after implementation the ENPs saw 2925 patients. This is $10.9 \%$ of all new patients attending the $A \& E$ department in the same period. Four hundred and fifty five patients were given drugs (either single doses or a course of treatment) by the ENPs. Two hundred and eighty eight drug courses were provided to take home and 167 single doses were given within the department. Therefore $15.5 \%$ of patients assessed and treated by ENPs received drugs according to the departmental protocols. Details of the drugs given are shown in fig 1 . Audit revealed no breaches of the protocols.

The audit highlighted the importance of documenting any decision not to give the medication, as well as thorough documenta- 
Drugs given by ENPs, Feb-Jul 1996

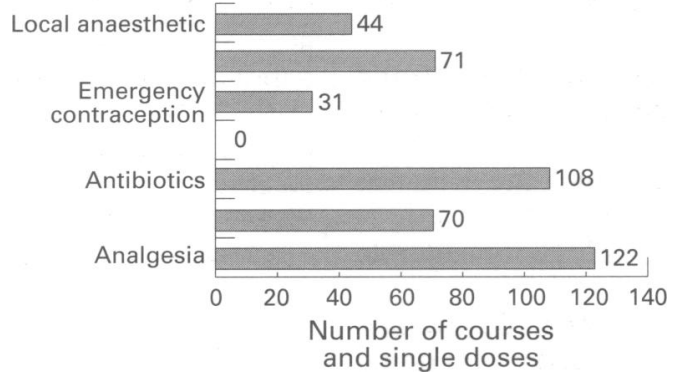

Figure 1 Breakdown of drugs (both courses and single doses) given by emergency nurse practitioners (ENPs) between February and fuly 1996.

tion of patients' current medication, allergies, and any contraindications.

In addition to the general audit of drug administration, the use of some specific drugs has undergone audit. The first was the administration of tetanus toxoid and tetanus immunoglobulin by the ENPs. The audit reviewed documentation of tetanus immunisation status, the appropriate use of tetanus toxoid, and tetanus immunoglobulin in tetanus-prone wounds. Results are shown in table 2 .

This audit resulted in minor changes to the protocol, for example an acknowledgement that it was acceptable practice to refer patients who thought they had received tetanus immunisation within the previous 10 years but were unsure of the exact date back to their general practitioner for review of their records.

The second specific audit we undertook was of the administration of postcoital contraception to patients attending the $A \& E$ department. The specific standards which were audited were documentation of unprotected intercourse less than 72 hours previously, documentation of a negative urine pregnancy test, documentation of no contraindications in the medical history, and documentation that the patient was not taking any drugs which may interact with the postcoital contraception. These standards reflect only part of the department protocol which includes the requirement to give advice concerning vomiting, follow up appointments, etc. There is a requirement for the patient to receive, read, and understand a postcoital contraception advice sheet before they leave the department.

This audit showed good compliance with the protocol (table 3). However, one ENP required further guidance in the appropriate documentation, particularly in relation to past medical history and potential drug interactions.

Audit has shown the value of ENPs having authority to give drugs to patients without reference to a doctor. In addition we have highlighted areas where the protocols required clarification and where individuals required further advice in interpretation of the protocol. Thus the audit process supports a dynamic approach to our protocols and training.

\section{Patient acceptability}

No patients have refused to accept medication from the ENPs, although the option to see a doctor is always available.
Table 2 Audit of tetanus immunisation administration

\begin{tabular}{lll}
\hline Standard & $\begin{array}{c}\text { Records } \\
\text { audited }\end{array}$ & Compliance \\
\hline $\begin{array}{l}\text { Documentation of tetanus } \\
\text { immunisation statistics }\end{array}$ & 50 & $94 \%$ \\
$\begin{array}{c}\text { Appropriate administration of } \\
\text { tetanus toxoid }\end{array}$ & 50 & $94 \%$ \\
$\begin{array}{c}\text { Appropriate administration of } \\
\text { tetanus immunoglobulin for } \\
\text { tetanus-prone wound }\end{array}$ & 8 & $100 \%$ \\
\hline
\end{tabular}

Table 3 Audit of emergency contraception administration

\begin{tabular}{llc}
\hline Standard & $\begin{array}{c}\text { Records } \\
\text { audited }\end{array}$ & Compliance \\
\hline $\begin{array}{c}\text { Documented negative pregnancy } \\
\text { test }\end{array}$ & 21 & $95 \%$ \\
$\begin{array}{c}\text { Unprotected intercourse }<72 \mathrm{~h} \\
\text { previously }\end{array}$ & 21 & $100 \%$ \\
$\begin{array}{c}\text { Documented relevant past } \\
\text { medical history }\end{array}$ & 21 & $71 \%$ \\
$\begin{array}{c}\text { Documented possible interactive } \\
\text { medication }\end{array}$ & 21 & $71 \%$ \\
\hline
\end{tabular}

\section{Discussion}

Shortly after the introduction of ENPs we became aware that their activities were limited by the requirement to find a doctor to prescribe drugs required by patients. This led to further delays for the patient, frustration for the ENPs, and inappropriate use of the doctor's time, who invariably felt they should re-evaluate the patient personally before prescribing.

In tackling this issue there were three main areas to be addressed: first, the legality of a nurse giving a drug against a protocol; second, the professional acceptability to nurses, doctors, and pharmacists of such an arrangement; and finally, the local attitudes of doctors, nurses, pharmacists, and the Trust Board, particularly in relation to vicarious liability. Extensive consultation showed that there were no obstacles to a nurse giving a drug to a patient according to a clearly defined protocol providing that protocol met all the requirements of the UKCC.

We recognise that others have reservations about the legality of schemes such as ours. ${ }^{89}$ The legal and professional opinion we have received is that it is reasonable to interpret "directions", as required by Section 58 (2) of the Medicines Act 1968, as covering "group protocols." Others take a different view; however, a debate as to which interpretation is right will remain one of "opinion" until the arguments are tested in the courts. In the meantime we have concluded that current legislation does not prohibit nurse administration of drugs according to protocols.

The whole A\&E team has found the implementation of the system beneficial. Our ENPs can deliver holistic care to the patient appropriate to their role as independent practitioners. This has increased the job satisfaction of the nurses who perform this role. Further, the delays patients experienced in receiving medication and care have been reduced, and the ENPs feel they are no longer waiting for doctors to be available to evaluate patients and sign prescriptions. The doctors are able to concentrate on those patients who require their 
particular skills and expertise without the distraction of writing unnecessary prescriptions.

Clinicians involved in such schemes must be aware that in the view of the GMC they remain ultimately responsible for the care delivered. It is therefore essential that the consultants and nurses work together and are actively involved in the development of protocols for the administration of drugs.

We would suggest that consultants are involved in the training and accreditation of the nurses who will give the drugs. If consultants are to remain responsible for care, we believe it is essential that they participate jointly with the nurses in a continuing audit programme to ensure that the protocols are followed, and cooperate in any revisions or alterations to those protocols.

We would not support consultants abdicating responsibility for the appropriate administration of drugs once the protocol has been agreed, although some have argued that if a clinician and nurse develop an acceptable protocol, and the clinician is satisfied that the nurse has undergone appropriate training and understands the protocol, then the nurse is solely accountable if he or she departs from that protocol. $^{10}$

We should have a dynamic approach to protocols. Ambiguous areas and the need for revision and review become clear when the protocol is in use. Problems will only be revealed by regular audit. Further, review of individual knowledge and understanding must continue to ensure there are no differences in interpretation or misunderstandings of the protocols.

The success of the scheme in the $A \& E$ department has prompted other clinical areas within our Trust to explore nurse administration of drugs according to protocol. Schemes have been successfully implemented within ophthalmology and family planning. Nurses on our coronary care unit are hoping to develop protocols for fast track thrombolysis with streptokinase. Within the A\&E department we see the scheme developing into two ways. First, we shall explore extending the drugs available to include eye preparations and the intramuscular administration of some non-steroidal anti-inflammatory drugs. Second, we wish to extend the scheme to level 1 nurses who undertake triage, so that patients can receive certain drugs, for example simple analgesia, immediately after triage.

We have improved the quality of service to patients in our A\&E department by the development of ENPs with the authority to give a wide range of drugs according to protocols. We believe that our careful analysis of the legal and professional issues in this area has been worthwhile. Our multidisciplinary A\&E team, including pharmacy colleagues, is involved in the development of protocols and audit. We have safely enhanced the quality of care given to patients attending our $A \& E$ department.

This work would not have been possible without the efforts of the Norfolk \& Norwich Health Care NHS Trust drugs and therapeutics committee and particularly Rick Adams (head of clinical pharmacy), David Carrington (director of pharmacy), Richard Drew (director of administration), Susan Henry (then nursing development manager), and Dr Magdi Naguib (chairman). We are grateful to Bruce Finlayson and Keith Walters (consultants in accident and emergency medicine), for their work in developing the protocols and in training. Finally, this initiative would not have been successful without the enthusiasm and commitment of the emergency nurse practitioners themselves.

Medicines Act 1968. London: HMSO, reprinted 1986.

2 United Kingdom Central Council for Nursing Midwifery and Health Visiting. Code of professional conduct. London: UKCC, 1992.

3 United Kingdom Central Council for Nursing, Midwifery and Health Visiting. Standards for the administration of medicines. London: UKCC, 1992.

4 United Kingdom Central Council for Nursing, Midwifery and Health Visiting. The scope of professional practice. and Health Visiting.

5 United Kingdom Central Council for Nursing, Midwifery and Health Visiting. Rule 18 (1) of the Nurses, Midwives \& Health Visitors Rules Approval order No 873. London: UKCC, 1983.

6 United Kingdom Central Council for Nursing, Midwifery and Health Visiting. Exercising accountability. London: UKCC, 1989.

7 General Medical Council. Professional Conduct and Discipline. Fitness to practise. Guidance from the General Medical Council. London: GMC, 1993.

8 Peysner J. Nurse prescribers. J Med Defence Union 1996;12(2):38-9.

9 Royal College of Nursing. Supply and administration of prescription only medicine. London: RCN, 1996.

10 Dowling S, Martin R, Skidmore P, Doyal L, Cameron A, Lloyd S. Nurses taking on junior doctors' work: a confusion of accountability. BMJ 1996;312:1211-4. 